Bull. Korean Math. Soc. 49 (2012), No. 6, pp. 1327-1334

http://dx.doi.org/10.4134/BKMS.2012.49.6.1327

\title{
AN IDENTITY ON THE 2m-TH POWER MEAN VALUE OF THE GENERALIZED GAUSS SUMS
}

\author{
Feng LiU AND Quan-Hui Yang
}

\begin{abstract}
In this paper, using analytic method and the properties of the Legendre's symbol, we prove an exact calculating formula on the $2 m$ th power mean value of the generalized quadratic Gauss sums for $m \geq 2$. This solves a conjecture of He and Zhang [On the $2 k$-th power mean value of the generalized quadratic Gauss sums, Bull. Korean Math. Soc. 48 (2011), no. 1, 9-15].
\end{abstract}

\section{Introduction}

Let $q \geq 2$ be an integer and $\chi$ be a Dirichlet character modulo $q$. For any integer $n$, the classical quadratic Gauss sums $G(n ; q)$ and the generalized quadratic Gauss sums $G(n, \chi ; q)$ are defined respectively by

$$
G(n ; q)=\sum_{a=1}^{q} e\left(\frac{n a^{2}}{q}\right),
$$

and

where $e(x)=e^{2 \pi i x}$.

$$
G(n, \chi ; q)=\sum_{a=1}^{q} \chi(a) e\left(\frac{n a^{2}}{q}\right)
$$

The study of $G(n, \chi ; q)$ is important in number theory, since it is a generalization of $G(n, q)$. In [4], Weil proved that if $p \geq 3$ is a prime, then

$$
|G(n, \chi ; p)| \leq 2 \sqrt{p} .
$$

In fact, Cochrane and Zheng [2] generalized this result to any integer. That is, for any integer $n$ with $(n, q)=1$, we have

$$
|G(n, \chi ; q)| \leq 2^{\omega(q)} \sqrt{q},
$$

Received June 30, 2011; Revised August 22, 2011.

2010 Mathematics Subject Classification. Primary 11L05; Secondary 11M20.

Key words and phrases. $2 m$-th power mean, exact calculating formula, generalized quadratic Gauss sums.

This work was supported by the National Natural Science Foundation of China, Grant No. 11071121.

(C)2012 The Korean Mathematical Society 
where $\omega(q)$ is the number of all distinct prime divisors of $q$.

Beside the upper bound of $G(n, \chi ; q)$, the power mean value of $|G(n, \chi ; q)|$ had also been studied by some authors. W. Zhang (see [5]) proved that if $p$ is an odd prime and $n$ is an integer with $(n, p)=1$, then

$$
\sum_{\chi}|G(n, \chi ; p)|^{4}= \begin{cases}(p-1)\left[3 p^{2}-6 p-1+4\left(\frac{n}{p}\right) \sqrt{p}\right], & \text { if } p \equiv 1 \bmod 4 ; \\ (p-1)\left(3 p^{2}-6 p-1\right), & \text { if } p \equiv 3 \bmod 4\end{cases}
$$

and

$$
\sum_{\chi}|G(n, \chi ; p)|^{6}=(p-1)\left(10 p^{3}-25 p^{2}-4 p-1\right), \text { if } p \equiv 3 \bmod 4,
$$

where $\left(\frac{n}{p}\right)$ is the Legendre symbol. For $p \equiv 1 \bmod 4$, it is still an open problem to calculate the exact value of $\sum_{\chi} \bmod p|G(n, \chi ; p)|^{6}$.

In 2005, W. Zhang and H. Liu [6] proved that if $q \geq 3$ is a square-full number, then for any integer $n, k$ with $(n k, q)=1, k \geq 1$, we have

$$
\sum_{\bmod q}|G(n, k, \chi ; q)|^{4}=q \cdot \phi^{2}(q) \prod_{p \mid q}(k, p-1)^{2} \cdot \prod_{\substack{p \mid q \\(k, p-1)=1}} \frac{\phi(p-1)}{p-1}
$$

where $\phi(q)$ is the Euler function and $G(n, k, \chi ; q)=\sum_{a=1}^{q} \chi(a) e\left(\frac{n a^{k}}{q}\right)$.

Recently, Y. He and W. Zhang [3] proved the following result.

Let odd number $q>1$ be a square-full number. Then for any integer $n$ with $(n, q)=1$ and $k=3$ or 4 , we have the identity

$$
\sum_{\chi}|G(n, \chi ; q)|^{2 k}=4^{(k-1) \omega(q)} \cdot q^{k-1} \cdot \phi^{2}(q) .
$$

Besides, they conjectured the above identity also holds for $k \geq 5$.

In this paper, we prove this conjecture in the following theorem.

Theorem 1. Let odd number $q>1$ be a square-full number, $m \geq 2$ be an integer. Then for any integer $n$ with $(n, q)=1$, we have the identity

$$
\sum_{\chi}|G(n, \chi ; q)|^{2 m}=4^{(m-1) \omega(q)} \cdot q^{m-1} \cdot \phi^{2}(q) .
$$

\section{Proofs}

Lemma 1 (See [1, Theorem 9.13]). If $p$ is an odd prime, then we have

$$
\left(\sum_{a=1}^{p-1}\left(\frac{a}{p}\right) e\left(\frac{a}{p}\right)\right)^{2}=\left(\frac{-1}{p}\right) p .
$$

Lemma 2. For any odd prime $p$, we have

$$
G^{2}(1 ; p)=\left(\frac{-1}{p}\right) p .
$$


Proof. This is a corollary of Lemma 1 in [3].

Let $p \geq 3$ be a prime, and let $k, n, a$ be three integers with $1 \leq k \leq n$. Write

$$
T_{p}(n, k, a)=\sum_{\substack{x_{1}=1 \\ x_{1}+x_{2}+\cdots+x_{n} \equiv a}}^{p-1} \sum_{\substack{x_{2}=1 \\ x_{n}=1 \\ \bmod p}}^{p-1}\left(\frac{x_{1} x_{2} \cdots x_{k}}{p}\right) .
$$

In order to prove Theorem 1 , we need a lemma on the value of $T_{p}(n, k, a)$.

Lemma 3. Let $p \geq 3$ be a prime, and let $k, n$, a be three integers with $1 \leq k \leq$ $n$. Then we have

$$
T_{p}(n, k, a)= \begin{cases}(-1)^{n-k}\left(\frac{a}{p}\right) p^{(k-1) / 2}\left(\frac{-1}{p}\right)^{(k-1) / 2}, & \text { if } 2 \nmid k \text { and } p \nmid a ; \\ 0, & \text { if } 2 \nmid k \text { and } p \mid a ; \\ (-1)^{n+1-k}\left(\frac{-1}{p}\right)^{k / 2} p^{(k-2) / 2}, & \text { if } 2 \mid k \text { and } p \nmid a ; \\ (-1)^{n-k}(p-1)\left(\frac{-1}{p}\right)^{k / 2} p^{(k-2) / 2}, & \text { if } 2 \mid k \text { and } p \mid a ;\end{cases}
$$

and

(2) $\quad \sum_{\substack{x_{1}=1 \\ x_{1}+x_{2}+\cdots+x_{n} \equiv a}}^{p-1} \cdots \sum_{\substack{x_{n}=1 \\ \bmod p}}^{p-1} 1= \begin{cases}\left((p-1)^{n}-(-1)^{n}\right) / p, & \text { if } p \nmid a ; \\ \left((p-1)^{n}+(p-1)(-1)^{n}\right) / p, & \text { if } p \mid a .\end{cases}$

Proof. For any $1 \leq k \leq n$, we have

$$
\begin{aligned}
& T_{p}(n, k, a)=\sum_{\substack{x_{1}=1 \\
x_{1}+x_{2}+\cdots+x_{n} \equiv a \bmod p}}^{p-1} \cdots \sum_{x_{n}=1}^{p-1}\left(\frac{x_{1} x_{2} \cdots x_{k}}{p}\right) \\
= & \frac{1}{p} \sum_{x_{1}=1}^{p-1} \cdots \sum_{x_{n}=1}^{p-1}\left(\frac{x_{1} x_{2} \cdots x_{k}}{p}\right) \sum_{m=1}^{p} e\left(\frac{m\left(x_{1}+x_{2}+\cdots+x_{n}-a\right)}{p}\right) \\
= & \frac{1}{p} \sum_{m=1}^{p-1} e\left(\frac{-m a}{p}\right)\left(\sum_{x=1}^{p-1}\left(\frac{x}{p}\right) e\left(\frac{m x}{p}\right)\right)^{k}\left(\sum_{y=1}^{p-1} e\left(\frac{m y}{p}\right)\right)^{n-k} \\
= & \frac{(-1)^{n-k}}{p} \sum_{m=1}^{p-1}\left(\frac{m}{p}\right)^{k} e\left(\frac{-m a}{p}\right)\left(\sum_{x=1}^{p-1}\left(\frac{x}{p}\right) e\left(\frac{x}{p}\right)\right)^{k} .
\end{aligned}
$$

Case 1. $p \mid a, 2 \nmid k$. Then by (3) we have

$$
T_{p}(n, k, a)=\frac{(-1)^{n-k}}{p}\left(\sum_{x=1}^{p-1}\left(\frac{x}{p}\right) e\left(\frac{x}{p}\right)\right)^{k}\left(\sum_{m=1}^{p-1}\left(\frac{m}{p}\right)\right)=0 .
$$


Case 2. $p|a, 2| k$. Then by Lemma 1 and (3), we have

$$
\begin{aligned}
T_{p}(n, k, a) & =\frac{(-1)^{n-k}}{p} \sum_{m=1}^{p-1}\left(\frac{-1}{p}\right)^{k / 2} p^{k / 2} \\
& =(-1)^{n-k}(p-1)\left(\frac{-1}{p}\right)^{k / 2} p^{(k-2) / 2} .
\end{aligned}
$$

Case 3. $p \nmid a, 2 \nmid k$. Then by Lemma 1 and (3), we have

$$
\begin{aligned}
& T_{p}(n, k, a) \\
= & \frac{(-1)^{n-k}}{p}\left(\frac{-a}{p}\right) \sum_{m=1}^{p-1}\left(\frac{m}{p}\right) e\left(\frac{m}{p}\right)\left(\sum_{x=1}^{p-1}\left(\frac{x}{p}\right) e\left(\frac{x}{p}\right)\right)^{k} \\
= & \frac{(-1)^{n-k}}{p}\left(\frac{-a}{p}\right)\left(\frac{-1}{p}\right)^{(k+1) / 2} p^{(k+1) / 2} \\
= & (-1)^{n-k}\left(\frac{a}{p}\right) p^{(k-1) / 2}\left(\frac{-1}{p}\right)^{(k-1) / 2} .
\end{aligned}
$$

Case 4. $p \nmid a, 2 \mid k$. Then by Lemma 1 and (3), we have

$$
\begin{aligned}
& T_{p}(n, k, a) \\
= & \frac{(-1)^{n-k}}{p}\left(\frac{-1}{p}\right)^{k / 2} p^{k / 2} \sum_{m=1}^{p-1} e\left(\frac{-m a}{p}\right) \\
= & (-1)^{n+1-k}\left(\frac{-1}{p}\right)^{k / 2} p^{(k-2) / 2} .
\end{aligned}
$$

Next, we shall prove the equality (2) in Lemma 3.

(4)

$$
\begin{aligned}
& \sum_{\substack{x_{1}=1 \\
x_{1}+x_{2}+\cdots+x_{n} \equiv a}}^{p-1} \cdots \sum_{\substack{x_{n}=1 \\
\bmod p}}^{p-1} 1 \\
= & \frac{1}{p} \sum_{x_{1}=1}^{p-1} \sum_{x_{2}=1}^{p-1} \cdots \sum_{x_{n}=1}^{p-1} \sum_{m=1}^{p} e\left(\frac{m\left(x_{1}+x_{2}+\cdots+x_{n}-a\right)}{p}\right) \\
= & \frac{1}{p} \sum_{m=1}^{p} e\left(\frac{-m a}{p}\right)\left(\sum_{x=1}^{p-1} e\left(\frac{m x}{p}\right)\right)^{n} \\
= & \frac{1}{p} \sum_{m=1}^{p-1} e\left(\frac{-m a}{p}\right)(-1)^{n}+\frac{(p-1)^{n}}{p} .
\end{aligned}
$$

If $p \mid a$, then the right side of (4) becomes $\left((p-1)^{n}+(p-1)(-1)^{n}\right) / p$; if $p \nmid a$, then the right side of $(4)$ becomes $\left((p-1)^{n}-(-1)^{n}\right) / p$.

This completes the proof of Lemma 3. 
Lemma 4. Let $p \geq 3$ be a prime, $\alpha \geq 2$, a and $n$ be three integers with $1 \leq a \leq p^{\alpha}-1$ and $(n, p)=1$. If $p^{\alpha-1} \| \bar{a}^{2}-1$, we write $a=r p^{\alpha-1}+\varepsilon$, where $1 \leq r \leq p-1$ and $\varepsilon= \pm 1$, then we have

$$
\sum_{b=1}^{p^{\alpha}} e\left(\frac{n b^{2}\left(a^{2}-1\right)}{p^{\alpha}}\right)= \begin{cases}0, & \text { if } p^{\alpha-1} \nmid a^{2}-1 ; \\ p^{\alpha-1}\left[\left(\frac{2 \varepsilon r n}{p}\right) G(1 ; p)-1\right], & \text { if } p^{\alpha-1} \| a^{2}-1 ; \\ \phi\left(p^{\alpha}\right), & \text { if } p^{\alpha} \mid a^{2}-1\end{cases}
$$

Proof. See the proof of Lemma 4 of [3].

Lemma 5 (See [6, Lemma 6]). Let $m, n \geq 2$ and $u$ be three integers with $(m, n)=1$ and $(u, m n)=1$. Then for any character $\chi=\chi_{1} \chi_{2}$ with $\chi_{1} \bmod m$ and $\chi_{2} \bmod n$, we have the identity

$$
G(u, \chi ; m n)=\chi_{1}(n) \chi_{2}(m) G\left(u n, \chi_{1} ; m\right) G\left(u m, \chi_{2} ; n\right) .
$$

Lemma 6. Let $p \geq 3$ be a prime, $\alpha \geq 2, m \geq 2$ be two integers. Then for any integer $n$ with $(n, p)=1$, we have the identity

$$
\sum_{\chi}\left|G\left(n, \chi ; p^{\alpha}\right)\right|^{2 m}=4^{(m-1)} \cdot \phi^{2}\left(p^{\alpha}\right) \cdot p^{(m-1) \alpha} .
$$

Proof. By the definition of $G\left(n, \chi ; p^{\alpha}\right)$, we have

$$
\begin{aligned}
\left|G\left(n, \chi ; p^{\alpha}\right)\right|^{2} & =\sum_{a=1}^{p^{\alpha}} \sum_{b=1}^{p^{\alpha}} \chi(a) \overline{\chi(b)} e\left(\frac{n\left(a^{2}-b^{2}\right)}{p^{\alpha}}\right) \\
& =\sum_{a=1}^{p^{\alpha}} \chi(a) \sum_{b=1}^{p^{\alpha}} e\left(\frac{n b^{2}\left(a^{2}-1\right)}{p^{\alpha}}\right) .
\end{aligned}
$$

Hence, by this formula we have

$$
\begin{aligned}
& \sum_{\chi \bmod p^{\alpha}}\left|G\left(n, \chi ; p^{\alpha}\right)\right|^{2 m} \\
= & \sum_{\chi} \sum_{\bmod p^{\alpha}}^{p^{\alpha} x_{1}=1 x_{2}=1} \sum^{\alpha} \cdots \sum_{x_{m}=1}^{p^{\alpha}} \chi\left(x_{1} \cdots x_{m}\right) \prod_{i=1}^{m}\left(\sum_{y_{i}=1}^{p^{\alpha}} e\left(\frac{n y_{i}^{2}\left(x_{i}^{2}-1\right)}{p^{\alpha}}\right)\right) \\
= & \phi\left(p^{\alpha}\right) \sum_{x_{1}=1 x_{2}=1}^{p^{\alpha}} \sum_{x_{1} x_{2} \cdots x_{m} \equiv 1}^{p^{\alpha}} \cdots \sum_{\substack{x_{m}=1 \\
\bmod p^{\alpha}}}^{\prime} \prod_{i=1}^{m}\left(\sum_{y_{i}=1}^{p^{\alpha}} e\left(\frac{n y_{i}^{2}\left(x_{i}^{2}-1\right)}{p^{\alpha}}\right)\right) .
\end{aligned}
$$

Then by Lemma 4 we have

$$
\sum_{\chi \bmod p^{\alpha}}\left|G\left(n, \chi ; p^{\alpha}\right)\right|^{2 m}=\phi\left(p^{\alpha}\right) \sum_{k=0}^{m}\left(\begin{array}{c}
m \\
k
\end{array}\right) A(m, k),
$$


where

$$
A(m, k)=\sum_{\substack{x_{1}=1 \\ p^{\alpha-1} \| x_{1}^{2}-1}}^{p^{\alpha}} \ldots \sum_{\substack{x_{k}=1 \\ p^{\alpha-1} \| x_{k}^{2}-1 p^{\alpha} \mid x_{k+1}^{2}-1 \\ x_{1} x_{2} \cdots x_{m} \equiv 1}}^{p^{\alpha}} \sum_{\substack{x_{k+1}=1 \\ \bmod p^{\alpha}}}^{p^{\alpha}} \cdots \sum_{\substack{x_{m}=1 \\ p^{\alpha} \mid x_{m}^{2}-1}}^{p^{\alpha}} \prod_{i=1}^{\prime}\left(\sum_{y_{i}=1}^{p^{\alpha}} e\left(\frac{n y_{i}^{2}\left(x_{i}^{2}-1\right)}{p^{\alpha}}\right)\right) .
$$

Now, in order to prove Lemma 6 , we need to calculate $A(m, k)$.

$$
\begin{aligned}
& A(m, k) \\
& =\sum_{\substack{x_{1}=1 \\
p^{\alpha-1} \| x_{1}^{2}-1}}^{p^{\alpha}} \cdots \sum_{\substack{x_{k}=1 \\
p^{\alpha-1} \| x_{k}^{2}-1}}^{p^{\alpha}} \sum_{\substack{x_{k+1}=1 \\
p^{\alpha} \mid x_{k+1}^{2}-1}}^{p^{\alpha}} \cdots \sum_{\substack{x_{m}=1 \\
p^{\alpha} \mid x_{m}^{2}-1}}^{p^{\alpha}} \prod_{i=1}^{m}\left(\sum_{y_{i}=1}^{p^{\alpha}} e\left(\frac{n y_{i}^{2}\left(x_{i}^{2}-1\right)}{p^{\alpha}}\right)\right)
\end{aligned}
$$

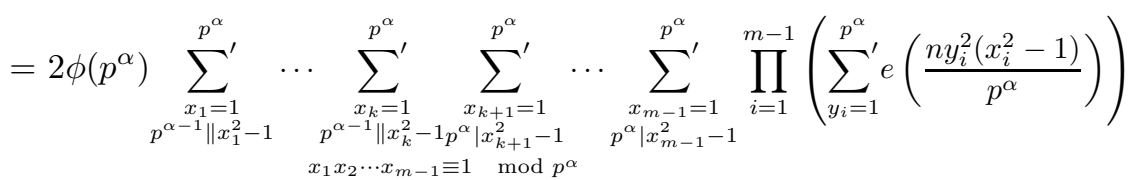

$$
\begin{aligned}
& =2 \phi\left(p^{\alpha}\right) A(m-1, k) .
\end{aligned}
$$

Hence, by induction on $m$, we have

$$
A(m, k)=2^{m-k} \phi^{m-k}\left(p^{\alpha}\right) A(k, k) .
$$

Next, we shall calculate $A(k, k)$. By the definition, we have

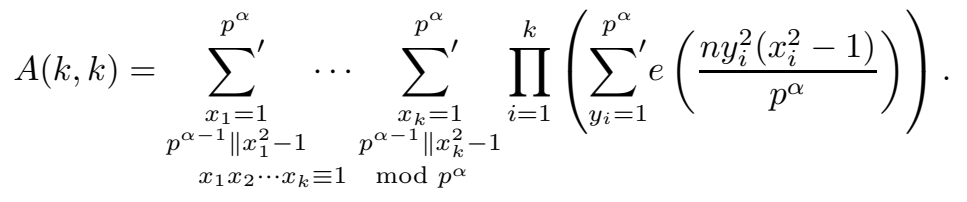

Write $x_{i}=r_{i} p^{\alpha-1}+\varepsilon_{i}\left(1 \leq r_{i} \leq p-1, \varepsilon_{i}= \pm 1\right)$ for $i=1,2, \ldots, k$. Then by Lemma 4 , we have

$$
\begin{aligned}
& A(k, k) \\
& =p^{k(\alpha-1)} \sum_{\substack{r_{1}=1 \\
\varepsilon_{1} r_{1}+\varepsilon_{2} r_{2}+\cdots+\varepsilon_{k} r_{k}=0 \\
\varepsilon_{1} \varepsilon_{2} \cdots \varepsilon_{k}=1}}^{p-1} \ldots \sum_{\substack{r_{k}=1 \\
\text { mod } p}}^{p-1} \prod_{i=1}^{k-1}\left(\left(\frac{2 n \varepsilon_{i} r_{i}}{p}\right) G(1 ; p)-1\right) \\
& =p^{k(\alpha-1)} \sum_{\substack{r_{1}=1 \\
r_{1}+r_{2}+\cdots+r_{k} \equiv 0 \\
r_{1} \varepsilon_{2} \cdots \varepsilon_{k}=1}}^{p-1} \cdots \sum_{r_{k}=1}^{p-1} \prod_{i=1}^{p-1}\left(\left(\frac{2 n r_{i}}{p}\right) G(1 ; p)-1\right) \\
& =2^{k-1} p^{k(\alpha-1)} \sum_{\substack{r_{1}=1 \\
r_{1}+r_{2}+\cdots+r_{k} \equiv 0}}^{p-1} \sum_{r_{2}=1}^{p-1} \sum_{r_{k}=1}^{p-1} \prod_{\substack{\bmod p \\
i=1}}^{k}\left(\left(\frac{2 n r_{i}}{p}\right) G(1 ; p)-1\right)
\end{aligned}
$$




$$
\begin{aligned}
= & 2^{k-1} p^{k(\alpha-1)} \cdot \sum_{\substack{r_{1}=1 \\
r_{1}+r_{2}+\cdots+r_{k} \equiv 0}}^{p-1} \cdots \sum_{\substack{r_{k}=1 \\
\bmod p}}^{p-1}\left((-1)^{k}\right. \\
& \left.+\sum_{j=1}^{k}(-1)^{k-j}\left(\begin{array}{c}
k \\
j
\end{array}\right)\left(\frac{2 n}{p}\right)^{j} G^{j}(1 ; p)\left(\frac{r_{1} r_{2} \cdots r_{j}}{p}\right)\right) .
\end{aligned}
$$

By Lemma 3, the above equality becomes

$$
\begin{aligned}
& A(k, k) \\
= & 2^{k-1} p^{k(\alpha-1)}(-1)^{k}\left(\frac{1}{p}\left((p-1)^{k}+(p-1)(-1)^{k}\right)\right. \\
& \left.+\sum_{j=1}^{\lfloor k / 2\rfloor}(-1)^{2 j}\left(\begin{array}{c}
k \\
2 j
\end{array}\right)\left(\frac{2 n}{p}\right)^{2 j} G^{2 j}(1 ; p)(-1)^{k-2 j}\left(\frac{-1}{p}\right)^{j} p^{j-1}(p-1)\right) .
\end{aligned}
$$

By Lemma 2, we have

$$
\begin{aligned}
& A(k, k) \\
= & 2^{k-1} p^{k(\alpha-1)-1}\left((-1)^{k}(p-1)^{k}+(p-1)+\sum_{j=1}^{\lfloor k / 2\rfloor}\left(\begin{array}{c}
k \\
2 j
\end{array}\right) p^{2 j}(p-1)\right) \\
= & 2^{k-1} p^{k(\alpha-1)-1}\left((-1)^{k}(p-1)^{k}+(p-1)\left((p+1)^{k}+(1-p)^{k}\right) / 2\right) \\
= & 2^{k-2} p^{k(\alpha-1)-1}\left((p+1)(1-p)^{k}+(p-1)(p+1)^{k}\right) .
\end{aligned}
$$

Hence, by (6) we have

$$
A(m, k)=2^{m-2} p^{m(\alpha-1)-1}\left((-1)^{k}(p+1)(p-1)^{m}+(p-1)^{m-k+1}(p+1)^{k}\right) .
$$

Finally, by (5) we have

$$
\begin{aligned}
& \sum_{\bmod p^{\alpha}}\left|G\left(n, \chi ; p^{\alpha}\right)\right|^{2 m} \\
= & \phi\left(p^{\alpha}\right) \sum_{k=0}^{m}\left(\begin{array}{c}
m \\
k
\end{array}\right) 2^{m-2} p^{m(\alpha-1)-1} \\
& \times\left((-1)^{k}(p+1)(p-1)^{m}+(p-1)^{m-k+1}(p+1)^{k}\right) \\
= & \phi\left(p^{\alpha}\right) 2^{m-2} p^{m(\alpha-1)-1}(p+1)(p-1)^{m} \sum_{k=0}^{m}\left(\begin{array}{c}
m \\
k
\end{array}\right)(-1)^{k} \\
& +\phi\left(p^{\alpha}\right) 2^{m-2} p^{m(\alpha-1)-1} \sum_{k=0}^{m}\left(\begin{array}{c}
m \\
k
\end{array}\right)(p-1)^{m-k+1}(p+1)^{k} \\
= & 0+\phi\left(p^{\alpha}\right) 2^{m-2} p^{m(\alpha-1)-1}(p-1)(2 p)^{m}
\end{aligned}
$$




$$
=4^{m-1} \phi^{2}\left(p^{\alpha}\right) p^{\alpha(m-1)} .
$$

This completes the proof of Lemma 6 .

Proof of Theorem 1. Since $q$ is an odd square-full number, let

$$
q=p_{1}^{\alpha_{1}} p_{2}^{\alpha_{2}} \cdots p_{\omega(q)}^{\alpha_{\omega(q)}}
$$

we have $\alpha_{i} \geq 2, i=1, \ldots, \omega(q)$. For any integer $n$ with $(n, q)=1$, by Lemma 5 and Lemma 6 , we obtain

$$
\begin{aligned}
\sum_{\bmod q}|G(n, \chi ; q)|^{2 m} & =\prod_{\substack{i=1 \\
p_{i}^{\alpha_{i}} \| q}}^{\omega(q)} \sum_{\bmod p_{i}^{\alpha_{i}}}\left|G\left(n q / p_{i}^{\alpha_{i}}, \chi ; p_{i}^{\alpha_{i}}\right)\right|^{2 m} \\
& =\prod_{\substack{i=1 \\
p_{i}^{\alpha_{i}} \| q}}^{\omega(q)}\left(4^{m-1} p_{i}^{\alpha_{i}(m-1)} \phi^{2}\left(p_{i}^{\alpha_{i}}\right)\right) \\
& =4^{(m-1) \omega(q)} \cdot q^{m-1} \cdot \phi^{2}(q) .
\end{aligned}
$$

This completes the proof of Theorem 1 .

Acknowledgement. We sincerely thank Professor Chun-Gang Ji for his valuable suggestions and useful discussions. We are also very grateful to Professor Wen-Peng Zhang for his very useful comments and helpful suggestions, especially for simplifying the proof of Lemma 3.

\section{References}

[1] Tom M. Apostol, Introduction to Analytic Number Theory, Spring-Verlag, New York, 1976.

[2] T. Cochrane and Z. Y. Zheng, Pure and mixed exponential sums, Acta Arith 91 (1999), no. 3, 249-278.

[3] Y. He and W. P. Zhang, On the $2 k$-th power mean value of the generalized quadratic Gauss sums, Bull. Korean Math. Soc. 48 (2011), no. 1, 9-15.

[4] A. Weil, On some exponential sums, Proc. Nat. Acad. Sci. U.S.A. 34 (1948), 204-207.

[5] W. P. Zhang, Moments of generalized quadratic Gauss sums weighted by L-functions, J. Number Theory 92 (2002), no. 2, 304-314.

[6] W. P. Zhang and H. Liu, On the general Gauss sums and their fourth power mean, Osaka J. Math. 42 (2005), no. 1, 189-199.

Feng LiU

School of Mathematical Sciences

NANJing Normal University

NANJiNG 210046, P. R. CHINA

E-mail address: fliu_19860126.com

QUAN-HUI YANG

School of Mathematical Sciences

Nanjing Normal University

NANJing 210046, P. R. China

E-mail address: yangquanhui01@163.com 